\title{
FUNCTIONAL CENTRAL LIMIT THEOREMS FOR MULTIVARIATE BESSEL PROCESSES IN THE FREEZING REGIME
}

\author{
MICHAEL VOIT, JEANNETTE H.C. WOERNER
}

\begin{abstract}
Multivariate Bessel processes $\left(X_{t, k}\right)_{t>0}$ describe interacting particle systems of Calogero-Moser-Sutherland type and are related with $\beta$-Hermite and $\beta$-Laguerre ensembles. They depend on a root system and a multiplicity $k$. Recently, several limit theorems were derived for $k \rightarrow \infty$ with fixed starting point. Moreover, the SDEs of $\left(X_{t, k}\right)_{t \geq 0}$ were used to derive strong laws of large numbers for $k \rightarrow \infty$ with starting points of the form $\sqrt{k} \cdot x$ with $x$ in the interior of the Weyl chambers. Here we provide associated almost sure functional central limit theorems which are locally uniform in $t$. The Gaussian limit processes admit explicit representations in terms of the solutions of associated deterministic ODEs.
\end{abstract}

\section{INTRODUCTION}

Integrable interacting particle systems on $\mathbb{R}$ of Calogero-Moser-Sutherland type with $N$ particles can be described as multivariate Bessel processes on appropriate closed Weyl chambers in $\mathbb{R}^{N}$. These processes are time-homogeneous diffusions with well-known generators of the transition semigroups and transition probabilities, and they are solution of the associated stochastic differential equations (SDEs); see [CGY, GY, R1, R2, RV1, RV2, DV, A]. These multivariate Bessel processes $\left(X_{t, k}\right)_{t \geq 0}$ are described via root systems, a possibly multidimensional multiplicity parameter $k$ and by their starting points $X_{0, k}:=x$. The multiplicies $k$ are coupling constants which describe the strength of interaction of the particles. We restrict our attention to the root systems $A_{N-1}, B_{N}$, and $D_{N}$ on $\mathbb{R}^{N}$ as the most relevant cases in view of interacting particle systems and random matrix theory.

We briefly recapitulate the most important cases $A_{N-1}$ and $B_{N}$. For $A_{N-1}$, we have a multiplicity $k \in] 0, \infty[$, the processes live on the closed Weyl chamber

$$
C_{N}^{A}:=\left\{x \in \mathbb{R}^{N}: \quad x_{1} \geq x_{2} \geq \ldots \geq x_{N}\right\},
$$

and the generator of the transition semigroup is

$$
L f:=\frac{1}{2} \Delta f+k \sum_{i=1}^{N}\left(\sum_{j \neq i} \frac{1}{x_{i}-x_{j}}\right) \frac{\partial}{\partial x_{i}} f,
$$

where we assume reflecting boundaries, i.e., the domain of $L$ is

$D(L):=\left\{\left.f\right|_{C_{N}^{A}}: f \in C^{(2)}\left(\mathbb{R}^{N}\right), \quad f\right.$ invariant under all coordinate permutations $\}$.

Date: October 21, 2019.

2010 Mathematics Subject Classification. Primary 60F15; Secondary 60F05, 60J60, 60B20, $60 \mathrm{H} 20,70 \mathrm{~F} 10,82 \mathrm{C} 22,33 \mathrm{C} 67$.

Key words and phrases. Interacting particle systems, Calogero-Moser-Sutherland models, multivariate Bessel processes, functional central limit theorems, random matrices. 
For $B_{N}$, we have the multiplicity $\left.k=\left(k_{1}, k_{2}\right) \in\right] 0, \infty\left[{ }^{2}\right.$, the processes live on

$$
C_{N}^{B}:=\left\{x \in \mathbb{R}^{N}: \quad x_{1} \geq x_{2} \geq \ldots \geq x_{N} \geq 0\right\},
$$

and the generator is

$$
L f:=\frac{1}{2} \Delta f+k_{2} \sum_{i=1}^{N} \sum_{j \neq i}\left(\frac{1}{x_{i}-x_{j}}+\frac{1}{x_{i}+x_{j}}\right) \frac{\partial}{\partial x_{i}} f \quad+k_{1} \sum_{i=1}^{N} \frac{1}{x_{i}} \frac{\partial}{\partial x_{i}} f,
$$

where we again assume reflecting boundaries.

By [R1, R2, RV1, RV2, the transition probabilities of $\left(X_{t, k}\right)_{t \geq 0}$ have the form

$$
K_{t}(x, A)=c_{k} \int_{A} \frac{1}{t^{\gamma_{k}+N / 2}} e^{-\left(\|x\|^{2}+\|y\|^{2}\right) /(2 t)} J_{k}\left(\frac{x}{\sqrt{t}}, \frac{y}{\sqrt{t}}\right) \cdot w_{k}(y) d y
$$

for $t>0, x \in C_{N}$, and $A \subset C_{N}$ a Borel set. For the root systems $A_{N-1}$ and $B_{N}$, we here have the weight functions $w_{k}$ of the form

$$
w_{k}^{A}(x):=\prod_{i<j}\left(x_{i}-x_{j}\right)^{2 k}, \quad w_{k}^{B}(x):=\prod_{i<j}\left(x_{i}^{2}-x_{j}^{2}\right)^{2 k_{2}} \cdot \prod_{i=1}^{N} x_{i}^{2 k_{1}},
$$

and $\gamma_{k}^{A}(k):=k N(N-1) / 2$ and $\gamma_{k}^{B}\left(k_{1}, k_{2}\right):=k_{2} N(N-1)+k_{1} N$ respectively. $w_{k}$ is homogeneous of degree $2 \gamma_{k}$, and $c_{k}>0$ is a known normalization, and $J_{k}$ a multivariate Bessel function of type $A_{N-1}$ or $B_{N}$ with multiplicities $k$ or $\left(k_{1}, k_{2}\right)$ which is analytic on $\mathbb{C}^{N} \times \mathbb{C}^{N}$ with $J_{k}(x, y)>0$ for $x, y \in \mathbb{R}^{N}$. Moreover, $J_{k}(x, y)=$ $J_{k}(y, x)$ and $J_{k}(0, y)=1$ for $x, y \in \mathbb{C}^{N}$. For this and further informations we refer e.g. to [R1, R2]. In particular, if $X_{0, k}=0$, then $X_{t, k}$ has the Lebesgue density

$$
\frac{c_{k}}{t^{\gamma+N / 2}} e^{-\|y\|^{2} /(2 t)} \cdot w_{k}(y) d y
$$

on $C_{N}$ for $t>0$. Hence, for the root systems $A_{N-1}, B_{N}$, these processes $\left(X_{t, k}\right)_{t \geq 0}$ (with proper parameters) are related to random matrix theory, as the distributions in (1.5) are those of the ordered eigenvalues of $\beta$-Hermite and $\beta$-Laguerre ensembles; see e.g. the tridiagonal random matrix models of Dumitriu and Edelman DE1, DE2. For other starting points, there exist further tridiagonal random matrix models for the distributions of $X_{t, k}$; see $\mathrm{AG}, \mathrm{HP}$.

We here study limit theorems for $\left(X_{t, k}\right)_{t \geq 0}$ when one or several components of $k$ tend to $\infty$ in a coupled way (we briefly write $k \rightarrow \infty$ by misuse of notation). In physics, this means freezing, and in random matrix theory, $k \rightarrow \infty$ roughly means $\beta \rightarrow \infty$ in DE2. Several limit theorems for $k \rightarrow \infty$ are known for fixed starting points $x \in \mathbb{R}^{N}$; see [AKM1, AKM2, AM, V] where for $x=0$ the results fit to [DE2]. In the present paper we regard the $\left(X_{t, k}\right)_{t \geq 0}$ as solutions of the SDEs

$$
d X_{t, k}=d B_{t}+\frac{1}{2}\left(\nabla\left(\ln w_{k}\right)\right)\left(X_{t, k}\right) d t
$$

with starting points $X_{0, k}=\sqrt{k} \cdot x$ with $x$ in the interior of the Weyl chambers and an $N$-dimensional Brownian motion $\left(B_{t}\right)_{t \geq 0}$. Note that by CGY], see also Sch] and [GM], under the condition $E\left(\int_{0}^{t} \nabla\left(\ln w_{k}\right)\left(X_{s, k}\right) d s\right)<\infty$, (1.6) has a unique (strong) solution $\left(X_{t, k}\right)_{t \geq 0}$, the corresponding Bessel process. Moreover, if all components of $k$ are at least $1 / 2$, and $x$ is in the interior of $C_{N}$, then $\left(X_{t, k}\right)_{t \geq 0}$ does not hit the boundary a.s..

The associated renormalized processes $\left(X_{t, k} / \sqrt{k}\right)_{t \geq 0}$ then start in $x$ and satisfy SDEs with drifts independent of $k$ and vanishing diffusion part as $k \rightarrow \infty$. In 
AV1, several strong, locally uniform limit theorems, of law of large number type, were derived. Moreover, only in a simple special case, a corresponding central limit theorem was derived in AV1. In the present paper we derive functional central limit theorems in general as follows: The renormalized processes $\left(X_{t, k} / \sqrt{k}\right)_{t \geq 0}$ satisfy SDEs with vanishing Brownian parts for $k \rightarrow \infty$. Let $\phi:=(\phi(t, x))_{t \geq 0}$ be the solution of the associated deterministic limit differential equation with start in $x$. We shall present Gaussian diffusions $W:=\left(W_{t}\right)_{t \geq 0}$ such that

$$
\sqrt{k}\left(\frac{X_{t, k}}{\sqrt{k}}-\phi(t, x)\right) \longrightarrow W_{t} \quad \text { for } \quad k \rightarrow \infty
$$

locally uniformly in $t$ with rate $O(1 / \sqrt{k})$ a.s.. This $\phi$ plays an essential role in these limit theorems, especially in the covariance matrix of $W$. Unfortunately, up to particular examples, $\phi$ cannot be written down explicitly. However, $\phi$ can be described explicitly in some new coordinates using elementary symmetric polynomials; we shall present these results on $\phi$ in $[\mathrm{VW}$. We mention that for particular starting points $x \in C_{N}$, the functions $\phi$ are provided explicitly via the zeros of the Hermite or Laguerre polynomial. This connection with zeros of classical orthogonal polynomials already appeared in [AKM1, AKM2, AM, AV1].

This paper is organized as follows. In the next two sections we study Bessel processes of type $A_{N-1}$ for $k \rightarrow \infty$ and, in the $B_{N}$-case, for multiplicities $k=$ $\left(k_{1}, k_{2}\right)=(\nu \cdot \beta, \beta)$ with $\nu>0$ and $\beta \rightarrow \infty$. Depending on $\nu$, here the zeros of the Laguerre polynomial $L_{N}^{\nu-1}$ play a prominent role. Section 4 is devoted to the root systems $D_{N}$. These results are then used in Section 5 to settle also the limits

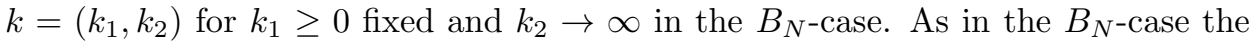
case $k=\left(k_{1}, k_{2}\right)$ for $k_{2} \geq 0$ fixed and $k_{1} \rightarrow \infty$ was already treated in [AV1, we skip this case here. In Section 6 we consider an extension of our results by adding an additional drift of the form $-\lambda X_{t, k}, \lambda \in \mathbb{R}$ to our SDEs. For $\lambda>0$ the resulting process is ergodic and mean reverting. For $N=1$ with $\lambda>0$, it is related to the Cox-Ingersoll-Ross process in finance.

\section{The Root System $A_{N-1}$}

The SDE (1.6) for Bessel processes $\left(X_{t, k}\right)_{t \geq 0}$ of type $A_{N-1}$ reads as

$$
d X_{t, k}^{i}=d B_{t}^{i}+k \sum_{j \neq i} \frac{1}{X_{t, k}^{i}-X_{t, k}^{j}} d t \quad(i=1, \ldots, N) .
$$

with an $N$-dimensional Brownian motion $\left(B_{t}^{1}, \ldots, B_{t}^{N}\right)_{t \geq 0}$. Hence, the renormalized processes $\left(\tilde{X}_{t, k}:=X_{t, k} / \sqrt{k}\right)_{t \geq 0}$ satisfy

$$
d \tilde{X}_{t, k}^{i}=\frac{1}{\sqrt{k}} d B_{t}^{i}+\sum_{j \neq i} \frac{1}{\tilde{X}_{t, k}^{i}-\tilde{X}_{t, k}^{j}} d t \quad(i=1, \ldots, N) .
$$

The solutions of (2.2) are closely related to limit $k=\infty$ in Lemma 2.1 of AV1. By AV1 we know that for each starting point $x_{0}$ in the interior of the Weyl chamber $C_{N}^{A}$, the dynamical system

with

$$
\frac{d x}{d t}(t)=H(x(t))
$$

$$
H(x):=\left(\sum_{j \neq 1} \frac{1}{x_{1}-x_{j}}, \ldots, \sum_{j \neq N} \frac{1}{x_{N}-x_{j}}\right)
$$


has a unique solution $\phi\left(t, x_{0}\right)$ for $t \geq 0$. It admits an explicit solution

$$
\phi(t, c \cdot z)=\sqrt{2 t+c^{2}} \cdot z
$$

for special starting points of the form $c z$, with $c>0$ and

$$
z:=\left(z_{1}, \ldots, z_{N}\right) \in C_{N}^{A}
$$

consisting of the ordered zeroes $z_{i}$ of Hermite polynomial $H_{N}$. We assume that the $\left(H_{N}\right)_{N \geq 0}$ are orthogonal w.r.t. the density $e^{-x^{2}}$ as in [S].

It can be shown that the solutions $\phi$ in (2.4) are attracting in some way, and all solutions of the ODEs (2.3) can be determined explicitly after some transformation of coordinates; we shall discuss this in VW].

We here only discuss the growth behavior of $\phi$. We observe that

$$
\begin{aligned}
\frac{d}{d t}\|\phi(t, x)\|_{2}^{2} & =2 \sum_{i=1}^{N} \phi_{i}(t, x) \cdot \dot{\phi}_{i}(t, x) \\
& =2 \sum_{i, j=1, \ldots, N, i \neq j} \frac{\phi_{i}(t, x)}{\phi_{i}(t, x)-\phi_{j}(t, x)}=N(N-1) .
\end{aligned}
$$

As $\|\phi(0, x)\|_{2}^{2}=\|x\|^{2}$, we see that for all $t \geq 0$ and all $x$,

$$
\|\phi(t, x)\|_{2}^{2}=N(N-1) t+\|x\|^{2} .
$$

We now use $\phi$ to derive limit theorems for Bessel processes of type $A_{N-1}$ for $k \rightarrow \infty$. We have the following strong limit law from Theorem 2.4 of AV1.

Theorem 2.1. Let $x$ be a point in the interior of $C_{N}^{A}$ and $y \in \mathbb{R}^{N}$. Let $k_{0} \geq 1 / 2$ such that $\sqrt{k} \cdot x+y$ is in the interior of $C_{N}^{A}$ for $k \geq k_{0}$.

For $k \geq k_{0}$ consider the Bessel processes $\left(X_{t, k}\right)_{t \geq 0}$ of type $A_{N-1}$ starting at $\sqrt{k} \cdot x+y$. Then, for all $t>0$,

$$
\sup _{0 \leq s \leq t, k \geq k_{0}}\left\|X_{s, k}-\sqrt{k} \phi(s, x)\right\|<\infty \quad \text { a.s.. }
$$

In particular, locally uniformly in $t$ a.s., $X_{t, k} / \sqrt{k} \rightarrow \phi(t, x)$ for $k \rightarrow \infty$.

We now turn to an associated functional central limit theorem. For this we again fix $x$ in the interior of $C_{N}^{A}$ and consider $t \mapsto \phi(t, x)(t \geq 0)$ as solution of (2.3). We also introduce the $N$-dimensional process $\left(W_{t}\right)_{t \geq 0}$ as unique solution of the inhomogeneous linear SDE

$$
d W_{t}^{i}=d B_{t}^{i}+\sum_{j \neq i} \frac{W_{t}^{j}-W_{t}^{i}}{\left(\phi_{i}(t, x)-\phi_{j}(t, x)\right)^{2}} d t \quad(i=1, \ldots, N) .
$$

with initial condition $W_{0}=0$; notice that the denominator is $\neq 0$ for $t>0$. In matrix notation, (2.9) means that

$$
d W_{t}=d B_{t}+A(t, x) W_{t} d t
$$

with the matrices $A(t, x) \in \mathbb{R}^{N \times N}$ with

$$
A(t, x)_{i, j}:=\frac{1}{\left(\phi_{i}(t, x)-\phi_{j}(t, x)\right)^{2}}, \quad A(t, x)_{i, i}:=-\sum_{j \neq i} \frac{1}{\left(\phi_{i}(t, x)-\phi_{j}(t, x)\right)^{2}}
$$


for $i, j=1, \ldots, N, i \neq j$. The process $\left(W_{t}\right)_{t \geq 0}$ is obviously Gaussian and admits the explicit representation in terms of matrix-valued exponentials

$$
W_{t}=e^{\int_{0}^{t} A(s, x) d s} \int_{0}^{t} e^{-\int_{0}^{s} A(u, x) d u} d B_{s} \quad(t \geq 0) .
$$

$\left(W_{t}\right)_{t \geq 0}$ is related to the Bessel processes $\left(X_{t, k}\right)_{t \geq 0}$ by the following functional CLT:

Theorem 2.2. Let $x$ be a point in the interior of $C_{N}^{A}$ and $y \in \mathbb{R}^{N}$. Let $k_{0} \geq 1 / 2$ such that $\sqrt{k} \cdot x+y$ is in the interior of $C_{N}^{A}$ for $k \geq k_{0}$. For $k \geq k_{0}$ consider the Bessel processes $\left(X_{t, k}\right)_{t \geq 0}$ of type $A_{N-1}$ starting at $\sqrt{k} \cdot x+y$. Then, for $t>0$,

$$
\sup _{0 \leq s \leq t, k \geq k_{0}} \sqrt{k} \cdot\left\|X_{s, k}-\sqrt{k} \phi(s, x)-W_{s}\right\|<\infty \quad \text { a.s. }
$$

i.e., $\lim _{k \rightarrow \infty} \sqrt{k}\left(\frac{X_{t, k}}{\sqrt{k}}-\phi(t, x)\right)=W_{t}$ locally uniformly in $t$ a.s. with rate $O(1 / \sqrt{k})$.

Proof. For $k \geq k_{0}$ consider the processes $\left(R_{t, k}:=X_{t, k}-\sqrt{k} \phi(t, x)-W_{t}\right)_{t \geq 0}$ on $\mathbb{R}^{N}$. Then $R_{0, k}=0$, and, by the SDEs (2.9) and (2.1) and the ODE for $\phi$ in (2.3),

$$
\begin{gathered}
R_{t, k}^{i}=k \int_{0}^{t} \sum_{j \neq i}\left(\frac{1}{X_{s, k}^{i}-X_{s, k}^{j}}-\frac{1}{\sqrt{k}\left(\phi_{i}(s, x)-\phi_{j}(s, x)\right)}\right. \\
\left.-\frac{W_{s}^{j}-W_{s}^{i}}{\left(\sqrt{k}\left(\phi_{i}(s, x)-\phi_{j}(s, x)\right)^{2}\right.}\right) d s
\end{gathered}
$$

for $i=1, \ldots, N$. We now use Taylor expansion for the function $1 / x$ with Lagrange remainder around some point $x_{0} \neq 0$, i.e.,

$$
\frac{1}{x}=\frac{1}{x_{0}}-\frac{x-x_{0}}{x_{0}^{2}}+\frac{\left(x-x_{0}\right)^{2}}{\tilde{x}^{3}}
$$

with some $\tilde{x}$ between $x \neq 0$ and $x_{0} \neq 0$ where $x, x_{0}$ have the same sign. Taking

$$
x=X_{s, k}^{i}-X_{s, k}^{j} \quad \text { and } \quad x_{0}=\sqrt{k}\left(\phi_{i}(s, x)-\phi_{j}(s, x)\right),
$$

we arrive at

$$
\begin{aligned}
R_{t, k}^{i} & =-\int_{0}^{t}\left(\sum_{j \neq i} \frac{\left(X_{s, k}^{i}-\sqrt{k} \phi_{i}(s, x)-W_{s}^{i}\right)-\left(X_{s, k}^{j}-\sqrt{k} \phi_{j}(s, x)-W_{s}^{j}\right)}{\left(\phi_{i}(s, x)-\phi_{j}(s, x)\right)^{2}}+H_{s, k}^{i}\right) d s \\
& =-\int_{0}^{t}\left(\sum_{j \neq i} \frac{R_{s, k}^{i}-R_{s, k}^{j}}{\left(\phi_{i}(s, x)-\phi_{j}(s, x)\right)^{2}}+H_{s, k}^{i}\right) d s
\end{aligned}
$$

with the error terms

$$
H_{s, k}^{i}=k \sum_{j \neq i} \frac{\left(\left(X_{s, k}^{i}-\sqrt{k} \phi_{i}(s, x)\right)-\left(X_{s, k}^{j}-\sqrt{k} \phi_{j}(s, x)\right)\right)^{2}}{\left(\sqrt{k}\left(\phi_{i}(s, x)-\phi_{j}(s, x)\right)+D_{i, j}(s)\right)^{3}}
$$

where, by the Lagrange remainder,

$$
\left|D_{i, j}(s)\right| \leq\left|\left(X_{s, k}^{j}-\sqrt{k} \phi_{j}(s, x)\right)-\left(X_{s, k}^{j}-\sqrt{k} \phi_{j}(s, x)\right)\right| .
$$

By Theorem 2.1, this can be bounded by some a.s. finite random variable $D$ independent of $i, j, s \in[0, t]$, and $k \geq k_{0}$ where $D$ depends on $x, y, t$. Therefore,

$$
\left|H_{s, k}^{i}\right| \leq H / \sqrt{k} \quad \text { for } \quad k \geq k_{0}, s \in[0, t], i=1, \ldots, N
$$


with some a.s. finite random variable $H$. In summary,

$$
R_{t, k}=-\int_{0}^{t}\left(A(s, x) R_{s, k}+H_{s, k}\right) d s, \quad R_{0, k}=0
$$

and thus, for suitable norms and all $u \in[0, t]$,

$$
\left\|R_{u, k}\right\| \leq A \int_{0}^{u}\left\|R_{s, k}\right\| d s+\frac{t \cdot\|H\|}{\sqrt{k}}
$$

with $A:=\sup _{s \in[0, t]}\|A(s, x)\|<\infty$. Hence, by the classical lemma of Gronwall,

$$
\left\|R_{u, k}\right\| \leq \frac{t\|H\|}{\sqrt{k}} e^{t A}
$$

for all $u \in[0, t]$. This yields the claim.

Remark 2.3. The processes $\left(X_{t, k}\right)_{t \geq 0}$ of type A admit some algebraic properties which are related with corresponding properties of $\phi$ and $A(t, x)$ :

(1) $\left(X_{t, k}\right)_{t \geq 0}$ has the same scaling as Brownian motions, i.e., for $r>0$, the process $\left(\frac{1}{r} X_{r^{2} t, k}\right)_{t \geq 0}$ is also a Bessel process of type A with the same $k$. The corresponding relations for $\phi$ and $A$ are

$\phi\left(r^{2} t, r x\right)=r \cdot \phi(t, x), \quad A\left(r^{2} t, r x\right)=\frac{1}{r^{2}} A(t, x) \quad$ for $\quad r>0, t \geq 0$.

Moreover, for $\left(W_{t}\right)_{t \geq 0}$ from (2.11), $\left(\frac{1}{r} W_{r^{2} t}\right)_{t \geq 0}$ is also a process of this type where $x$ is replaced by $r x$ in Eqs. (2.9) (2.11).

(2) By (2.1), the center of gravity

$$
\left(\overline{X_{t, k}}:=\frac{1}{N}\left(X_{t, k}^{1}+\ldots+X_{t, k}^{N}\right)=\frac{1}{N}\left(B_{t}^{1}+\ldots+B_{t}^{N}\right)\right)_{t \geq 0}
$$

is a Brownian motion up to scaling. For $\phi$ and $\left(W_{t}\right)_{t \geq 0}$ this means that

$$
\sum_{i=1}^{N} \phi_{i}(t, x)=x_{1}+\ldots+x_{N}, \quad W_{t}^{1}+\ldots+W_{t}^{N}=B_{t}^{1}+\ldots+B_{t}^{N}
$$

for $t \geq 0$. This yields that the sums over all rows and columns of $A(t, x)$ are equal to 0 , i.e., $A(t, x)$ is singular.

(3) Let $\left(\widehat{X_{t, k}}\right)_{t \geq 0}$ be the orthogonal projection of the Bessel process $\left(X_{t, k}\right)_{t \geq 0}$ to the orthogonal complement $(1, \ldots, 1)^{\perp} \subset \mathbb{R}^{N}$ of $(1, \ldots, 1)$. Then $\left(\widehat{X_{t, k}}\right)_{t \geq 0}$ is again a diffusion living on this $(N-1)$-dimensional subspace which is stochastically independent of the center-of-gravity-process $\left(\overline{X_{t, k}}\right)_{t \geq 0}$ on $\mathbb{R}$. $(1, \ldots, 1)$. On the level of $\phi$ and $A$, we have the relations

$$
\phi(t, x+r \cdot(1, \ldots, 1))=\phi(t, x)+r \cdot(1, \ldots, 1)
$$

and $A(t, x+r \cdot(1, \ldots, 1))=A(t, x)$ for $r \in \mathbb{R}$.

Remark 2.4. Similarly as in Theorem 2.2 we may deduce functional central limit theorems for the powers $X_{t, k}^{p}(p \in \mathbb{N})$ where these powers are taken in all coordinates. The most prominent examples appear for $p=2$. We have

$$
\sqrt{k}\left(\left(\frac{X_{t, k}}{\sqrt{k}}\right)^{p}-\phi^{p}(t, x)\right) \longrightarrow W_{t, p} \quad \text { for } \quad k \rightarrow \infty
$$

a.s. locally uniformly in $t$ with limit processes which solve

$$
d W_{t, p}=p \phi^{p-1}(t, x) d B_{t}+p A_{p}(t, x) W_{t, p} d t, \quad W_{0, p}=0
$$


with the matrices $A_{p}(t, x) \in \mathbb{R}^{N \times N}$ with

$$
\begin{gathered}
A_{p}(t, x)_{i, j}:=\frac{\phi_{i}^{p-1}(t, x)}{\left(\phi_{i}(t, x)-\phi_{j}(t, x)\right)^{2}} \quad \text { for } \quad i \neq j, \\
A_{p}(t, x)_{i, i}:=-\sum_{j \neq i} \frac{(p-2) \phi_{i}^{p-1}(t, x)-(p-1) \phi_{i}^{p-2}(t, x) \phi_{j}(t, x)}{\left(\phi_{i}(t, x)-\phi_{j}(t, x)\right)^{2}} .
\end{gathered}
$$

Remark 2.5. Assume now that $N$ is odd, and that $\left(X_{t, k}\right)_{t>0}$ starts at $\sqrt{k} c z$ in the interior of $C_{N}^{A}$. In order to study the $\frac{N-1}{2}+1$-th component, we notice that $z_{\frac{N-1}{2}+1}=0$ and that hence for $p \geq 2$ we have $W_{t}^{\frac{N-1}{2}+1}=0$ which implies a degenerate normal limiting distribution. This suggests that for the $\frac{N-1}{2}+1$-th component we have a faster rate of convergence than $\sqrt{k}$. Indeed from Theorem 2.2 we see that as $k \rightarrow \infty$

$$
X_{t, k}^{\frac{N-1}{2}+1} \stackrel{d}{\rightarrow} \mathcal{N}
$$

for some normal random variable $\mathcal{N}$ and hence $\left(X_{t, k}^{\frac{N-1}{2}+1}\right)^{p} \stackrel{d}{\rightarrow} \mathcal{N}^{p}$.

We finally calculate the covariance matrix of $W_{t}$ for the special solution $\phi$ given by (2.4). For this we introduce the matrix $A \in \mathbb{R}^{N \times N}$ with

$$
A_{i, i}:=-\sum_{j \neq i} \frac{1}{\left(z_{i}-z_{j}\right)^{2}}, \quad A_{i, j}:=\frac{1}{\left(z_{i}-z_{j}\right)^{2}} \quad \text { for } \quad i \neq j
$$

with $z$ as in (2.5). Moreover, let $E$ be the N-dimensional unit matrix. It is shown in AV2 that the matrix $E-A$ has the eigenvalues $1,2, \ldots, N$.

Lemma 2.6. Assume that $\left(X_{t, k}\right)_{t \geq 0}$ starts in the interior of $C_{N}^{A}$ in $\sqrt{k} \cdot c z+y$ with $y \in \mathbb{R}, z$ as in (2.5) and $c>0$. Then the covariance matrices $\Sigma_{t} \in \mathbb{R}^{N \times N}$ for $t>0$ of the limit process $\left(W_{t}\right)_{t \geq 0}$ are given by

$$
\Sigma_{t}=\left(t+\frac{c^{2}}{2}\right)(E-A)^{-1}\left(E-e^{\left(\ln \frac{c^{2}}{2 t+c^{2}}\right)(E-A)}\right) .
$$

with eigenvalues $\lambda_{k}^{A}(t, c)=\frac{1}{2 k} \frac{\left(2 t+c^{2}\right)^{k}-c^{2 k}}{\left(2 t+c^{2}\right)^{k-1}}(k=1, \ldots, N)$, where $\lambda_{1}^{A}(t, c)=t$.

Proof. For the special case $\phi(s, c z)=\sqrt{2 s+c^{2}} z$, the matrix function $A(s, c z)$ satisfies $A(s, c z)=\frac{1}{2 s+c^{2}} A$. Hence,

$$
W_{t}=e^{\left(\ln \left(2 t+c^{2}\right)-\ln c^{2}\right) A / 2} \int_{0}^{t} e^{\left(-\ln \left(2 s+c^{2}\right)+\ln c^{2}\right) A / 2} d B_{s} \quad(t \geq 0) .
$$

Since $A$ is real and symmetric with eigenvalues $1, \ldots, n$, we have $A=U D U^{t}$ with an orthogonal matrix $U$ and the diagonal matrix

$$
D=\operatorname{diag}\left(d_{1}, \ldots d_{N}\right):=\operatorname{diag}(0,-1,-2, \ldots,-N+1) .
$$

Hence,

$$
W_{t}=U \int_{0}^{t} \operatorname{diag}\left(\left(\frac{2 t+c^{2}}{2 s+c^{2}}\right)^{\frac{d_{1}}{2}}, \ldots,\left(\frac{2 t+c^{2}}{2 s+c^{2}}\right)^{\frac{d_{N}}{2}}\right) d \tilde{B}_{s} U^{t}
$$


with the rotated Brownian motion $\left(\tilde{B}_{t}:=U^{t} B_{t} U\right)_{t \geq 0}$. This, the Itô-isometry, and $d_{i} / 2 \neq 1$ for all $i$ yield

$$
\begin{aligned}
& \Sigma_{t}= U \cdot \int_{0}^{t} \operatorname{diag}\left(\left(\frac{2 t+c^{2}}{2 s+c^{2}}\right)^{\frac{d_{1}}{2}}, \ldots,\left(\frac{2 t+c^{2}}{2 s+c^{2}}\right)^{\frac{d_{N}}{2}}\right)^{2} d s \cdot U^{t} \\
&= U \cdot \int_{0}^{t} \operatorname{diag}\left(\left(\frac{2 t+c^{2}}{2 s+c^{2}}\right)^{d_{1}}, \ldots,\left(\frac{2 t+c^{2}}{2 s+c^{2}}\right)^{d_{N}}\right) d s \cdot U^{t} \\
&= U \cdot \operatorname{diag}\left(\frac{1}{2\left(1-d_{1}\right)}\left(2 t+c^{2}-c^{2\left(1-d_{1}\right)}\left(2 t+c^{2}\right)^{d_{1}}\right), \ldots,\right. \\
&\left.\frac{1}{2\left(1-d_{N}\right)}\left(2 t+c^{2}-c^{2\left(1-d_{N}\right)}\left(2 t+c^{2}\right)^{d_{N}}\right)\right) \cdot U^{t}
\end{aligned}
$$

As

$$
\left(U(E-D)^{-1} U^{t}\right)^{-1}=U(E-D) U^{t}=E-U D U^{t}=E-A
$$

and

$$
\frac{1}{2\left(1-d_{i}\right)}\left(2 t+c^{2}-c^{2\left(1-d_{i}\right)}\left(2 t+c^{2}\right)^{d_{i}}\right)=\frac{1}{1-d_{i}}\left(t+\frac{c^{2}}{2}\right)\left(1-e^{\left(1-d_{i}\right) \ln \frac{c^{2}}{2 t+c^{2}}}\right),
$$

we obtain by functional calculus that

$$
\begin{aligned}
\Sigma_{t} & =\left(t+\frac{c^{2}}{2}\right)(E-A)^{-1}\left(E-U \cdot \operatorname{diag}\left(e^{\left(1-d_{1}\right) \ln \frac{c^{2}}{2 t+c^{2}}}, \ldots, e^{\left(1-d_{N}\right) \ln \frac{c^{2}}{2 t+c^{2}}}\right) U^{t}\right) \\
& =\left(t+\frac{c^{2}}{2}\right)(E-A)^{-1}\left(E-e^{\left(\ln \frac{c^{2}}{2 t+c^{2}}\right)(E-A)}\right)
\end{aligned}
$$

which yields the desired form of the covariance matrix.

Remark 2.7. (1) The covariance matrix $(E-A)^{-1}$ above already appeared in $\mathrm{V}$ ] for the case $X_{0, k}=0$ and in [DE2] in the context of asymptotics for eigenvalues of Hermite ensembles. Note that though we assume $c>0$ we may formally set $c=0$ and obtain $\Sigma_{t}=t(E-A)^{-1}$ as in $[\mathrm{V}$. Since $\lim _{t \rightarrow \infty} \Sigma_{t} / t=(E-A)^{-1}$, we obtain asymptotically in $t$ the same result as starting in zero independent of the actual starting point $c z$.

(2) As

$$
\Sigma_{t}=\left(t+\frac{c^{2}}{2}\right)\left[-\ln \frac{c^{2}}{2 t+c^{2}} E-\frac{\left(\ln \frac{c^{2}}{2 t+c^{2}}\right)^{2}}{2}(E-A)-\cdots\right],
$$

the diagonal elements have a behavior in $t$ which is different from the remaining entries of $\Sigma_{t}$.

(3) Lemma 2.6 holds also for $t=0$ with $\Sigma_{0}=0$.

\section{The Root System $B_{N}$}

We now turn to Bessel processes for the root systems $B_{N}$ with multiplicities $k=\left(k_{1}, k_{2}\right)=(\nu \cdot \beta, \beta)$ with $\nu>0$ fixed and $\beta \rightarrow \infty$. We first recapitulate some facts from [AKM2] and [AV1]. The SDE (1.6) of type $B_{N}$ here reads as

$$
d X_{t, k}^{i}=d B_{t}^{i}+\beta \sum_{j \neq i}\left(\frac{1}{X_{t, k}^{i}-X_{t, k}^{j}}+\frac{1}{X_{t, k}^{i}+X_{t, k}^{j}}\right) d t+\frac{\nu \cdot \beta}{X_{t, k}^{i}} d t
$$


for $i=1, \ldots, N$ with an $N$-dimensional Brownian motion $\left(B_{t}^{1}, \ldots, B_{t}^{N}\right)_{t \geq 0}$. The renormalized processes $\left(\tilde{X}_{t, k}:=X_{t, k} / \sqrt{\beta}\right)_{t \geq 0}$ satisfy

$$
d \tilde{X}_{t, k}^{i}=\frac{1}{\sqrt{\beta}} d B_{t}^{i}+\sum_{j \neq i}\left(\frac{1}{\tilde{X}_{t, k}^{i}-\tilde{X}_{t, k}^{j}}+\frac{1}{\tilde{X}_{t, k}^{i}+\tilde{X}_{t, k}^{j}}\right) d t+\frac{\nu}{\tilde{X}_{t, k}^{i}} d t
$$

for $i=1, \ldots, N$. These processes are related with the deterministic limit case $\beta=\infty$; see Lemma 3.1 of AV1. By [AV1] we know that for $\nu>0$, for each starting point $x_{0}$ in the interior of $C_{N}^{B}$, the ODE

$$
\frac{d x}{d t}(t)=H(x(t))
$$

with

$$
H(x):=\left(\begin{array}{c}
\sum_{j \neq 1}\left(\frac{1}{x_{1}-x_{j}}+\frac{1}{x_{1}+x_{j}}\right)+\frac{\nu}{x_{1}} \\
\vdots \\
\sum_{j \neq N}\left(\frac{1}{x_{N}-x_{j}}+\frac{1}{x_{N}+x_{j}}\right)+\frac{\nu}{x_{N}}
\end{array}\right)
$$

has a unique solution $\phi\left(t, x_{0}\right)$ in the interior of $C_{N}^{B}$ for $t>0$. Furthermore, we obtain the special solution

$$
\phi(t, c \cdot y)=\sqrt{t+c^{2}} \cdot y
$$

for the special starting points $c y$, with $c>0$ and $y \in C_{N}^{B}$ given by the vector with

$$
\left(y_{1}^{2}, \ldots, y_{N}^{2}\right)=2\left(z_{1}^{(\nu-1)}, \ldots, z_{N}^{(\nu-1)}\right)
$$

Here $z_{1}^{(\nu-1)}, \ldots, z_{N}^{(\nu-1)}$ denote the ordered zeros of the Laguerre polynomials $L_{N}^{(\nu-1)}$.

For this we recapitulate that for $\alpha>0$ the Laguerre polynomials $\left(L_{n}^{(\alpha)}\right)_{n \geq 0}$ are orthogonal w.r.t. the density $e^{-x} \cdot x^{\alpha}$.

As in Section 2, the solutions $\phi$ in (3.4) are attracting in some way, and all solutions of the ODEs (3.3) can be determined explicitly after some transformation of coordinates; see [VW]. Moreover, similar to the preceding section,

$$
\begin{aligned}
\frac{d}{d t}\|\phi(t, x)\|^{2} & =2 \sum_{i=1}^{N} \phi_{i}(t, x) \cdot \dot{\phi}_{i}(t, x) \\
& =2 \sum_{i, j=1, \ldots, N, i \neq j}\left(\frac{\phi_{i}(t, x)}{\phi_{i}(t, x)-\phi_{j}(t, x)}+\frac{\phi_{i}(t, x)}{\phi_{i}(t, x)+\phi_{j}(t, x)}\right)+2 \nu N \\
& =2 N(N+\nu-1) .
\end{aligned}
$$

As $\|\phi(0, x)\|^{2}=\|x\|^{2}$, we obtain for $t \geq 0$ that

$$
\|\phi(t, x)\|^{2}=2 N(N+\nu-1) t+\|x\|^{2} .
$$

The solutions $\phi$ appear in the following strong limit law from AV1.

Theorem 3.1. Let $\nu>0$. Let $x$ be a point in the interior of $C_{N}^{B}$, and $y \in \mathbb{R}^{N}$. Let $\beta_{0} \geq 1 / 2$ with $\sqrt{\beta} \cdot x+y$ in the interior of $C_{N}^{B}$ for $\beta \geq \beta_{0}$. For $\beta \geq \beta_{0}$, consider the Bessel processes $\left(X_{t, k}\right)_{t \geq 0}$ of type $B$ with $k=\left(k_{1}, k_{2}\right)=(\beta \cdot \nu, \beta)$, which start in $\sqrt{\beta} \cdot x+y$. Then, for all $t>0$,

$$
\sup _{0 \leq s \leq t, \beta \geq \beta_{0}}\left\|X_{s, k}-\sqrt{\beta} \phi(s, x)\right\|<\infty \quad \text { a.s.. }
$$

In particular, $\lim _{\beta \rightarrow \infty} X_{t,(\nu \cdot \beta, \beta)} / \sqrt{\beta}=\phi(t, x)$ locally uniformly in $t$ a.s.. 
We now turn to an associated functional central limit theorem. We fix $x$ in the interior of $C_{N}^{B}$ and consider the associated $\phi(t, x)$. We also introduce the process $\left(W_{t}\right)_{t \geq 0}$ on $\mathbb{R}^{N}$ as unique solution of the inhomogeneous linear SDE

$$
\begin{aligned}
d W_{t}^{i}=d B_{t}^{i}+ & \sum_{j \neq i}\left(\frac{W_{t}^{j}-W_{t}^{i}}{\left(\phi_{i}(t, x)-\phi_{j}(t, x)\right)^{2}}-\frac{W_{t}^{j}+W_{t}^{i}}{\left(\phi_{i}(t, x)+\phi_{j}(t, x)\right)^{2}}\right) d t \\
& -\frac{\nu \cdot W_{t}^{i}}{\phi_{i}(t, x)^{2}} d t
\end{aligned}
$$

for $i=1, \ldots, N$ with initial condition $W_{0}=0$. Notice that all denominators are $\neq 0$ for $t>0$. (3.8) may be written in matrix notation as

$$
d W_{t}=d B_{t}+A_{\nu}(t, x) W_{t} d t
$$

with the matrices $A_{\nu}(t, x) \in \mathbb{R}^{N \times N}$ with

$$
\begin{aligned}
& A_{\nu}(t, x)_{i, j}:=\frac{1}{\left(\phi_{i}(t, x)-\phi_{j}(t, x)\right)^{2}}-\frac{1}{\left(\phi_{i}(t, x)+\phi_{j}(t, x)\right)^{2}} \quad(i \neq j), \\
& A_{\nu}(t, x)_{i, i}:=\sum_{j \neq i}\left(\frac{-1}{\left(\phi_{i}(t, x)-\phi_{j}(t, x)\right)^{2}}-\frac{1}{\left(\phi_{i}(t, x)+\phi_{j}(t, x)\right)^{2}}\right)-\frac{\nu}{\phi_{i}(t, x)^{2}}
\end{aligned}
$$

for $i, j=1, \ldots, N$. The process $\left(W_{t}\right)_{t \geq 0}$ is given by

$$
W_{t}=e^{\int_{0}^{t} A_{\nu}(s, x) d s} \int_{0}^{t} e^{-\int_{0}^{s} A_{\nu}(u, x) d u} d B_{s} \quad(t \geq 0) .
$$

This process is obviously Gaussian; we describe it more closely below.

Theorem 3.2. Let $\nu>0$. Let $x$ be a point in the interior of $C_{N}^{B}$ and let $y \in \mathbb{R}^{N}$. Let $\beta_{0} \geq 1 / 2$ such that $\sqrt{\beta} \cdot x+y$ is in the interior of $C_{N}^{A}$ for $\beta \geq \beta_{0}$.

For $\beta \geq \beta_{0}$ consider the Bessel processes $\left(X_{t, k}\right)_{t \geq 0}$ of type $B_{N}$ with $k=(\nu \beta, \beta)$ starting at $\sqrt{\beta} \cdot x+y$. Then, for all $t>0$,

$$
\sup _{0 \leq s \leq t, \beta \geq \beta_{0}} \sqrt{\beta} \cdot\left\|X_{s, k}-\sqrt{\beta} \phi(s, x)-W_{s}\right\|<\infty \quad \text { a.s. }
$$

i.e., $\lim _{\beta \rightarrow \infty} X_{s, k}-\sqrt{\beta} \phi(s, x)=W_{t}$ locally uniformly in $t$ a.s. with rate $O(1 / \sqrt{\beta})$.

Proof. For $\beta \geq \beta_{0}$ consider the processes

$$
\left(R_{t, \beta}:=X_{t,(\nu \beta, \beta)}-\sqrt{\beta} \phi(t, x)-W_{t}\right)_{t \geq 0}
$$

on $\mathbb{R}^{N}$ with $R_{0, \beta}=0$. Then by (3.8), (3.1) and the ODE (3.3), $R_{t, \beta}^{i}$ is eqaul to

$$
\begin{aligned}
\beta \int_{0}^{t} & {\left[\sum_{j \neq i}\left(\frac{1}{X_{s, k}^{i}-X_{s, k}^{j}}-\frac{1}{\sqrt{\beta}\left(\phi_{i}(s, x)-\phi_{j}(s, x)\right)}-\frac{W_{s}^{j}-W_{s}^{i}}{\left(\sqrt{\beta}\left(\phi_{i}(s, x)-\phi_{j}(s, x)\right)^{2}\right.}\right)\right.} \\
& +\sum_{j \neq i}\left(\frac{1}{X_{s, k}^{i}+X_{s, k}^{j}}-\frac{1}{\sqrt{\beta}\left(\phi_{i}(s, x)+\phi_{j}(s, x)\right)}+\frac{W_{s}^{j}+W_{s}^{i}}{\left(\sqrt{\beta}\left(\phi_{i}(s, x)+\phi_{j}(s, x)\right)^{2}\right.}\right) \\
& \left.+\nu\left(\frac{1}{X_{s, k}^{i}}-\frac{1}{\sqrt{\beta} \phi_{i}(s, x)}+\frac{W_{s}^{i}}{\left(\sqrt{\beta} \phi_{i}(s, x)\right)^{2}}\right)\right] d s .
\end{aligned}
$$

The Taylor expansion for $1 / x$ with Lagrange remainder at $x_{0} \neq 0$ yields

$$
1 / x=1 / x_{0}-\left(x-x_{0}\right) /\left(x_{0}^{2}\right)+\left(x-x_{0}\right)^{2} / \tilde{x}^{3}
$$


with some $\tilde{x}$ between $x \neq 0$ and $x_{0} \neq 0$ which have the same signs. Taking

$$
\begin{aligned}
x & =X_{s, k}^{i} \pm X_{s, k}^{j}, & x_{0} & =\sqrt{\beta}\left(\phi_{i}(s, x) \pm \phi_{j}(s, x)\right) \quad \text { and } \\
x & =X_{s, k}^{i}, & x_{0} & =\sqrt{\beta} \phi_{i}(s, x),
\end{aligned}
$$

we get

$$
\begin{aligned}
R_{t, \beta}^{i}=- & \int_{0}^{t}\left(\sum_{j \neq i} \frac{\left(X_{s, k}^{i}-\sqrt{\beta} \phi_{i}(s, x)-W_{s}^{i}\right)-\left(X_{s, k}^{j}-\sqrt{\beta} \phi_{j}(s, x)-W_{s}^{j}\right)}{\left(\phi_{i}(s, x)-\phi_{j}(s, x)\right)^{2}}\right. \\
& +\sum_{j \neq i} \frac{\left(X_{s, k}^{i}-\sqrt{\beta} \phi_{i}(s, x)-W_{s}^{i}\right)+\left(X_{s, k}^{j}-\sqrt{\beta} \phi_{j}(s, x)-W_{s}^{j}\right)}{\left(\phi_{i}(s, x)+\phi_{j}(s, x)\right)^{2}} \\
& \left.+\frac{X_{s, k}^{i}-\sqrt{\beta} \phi_{i}(s, x)-W_{s}^{i}}{\phi_{i}(s, x)^{2}}+H_{s, \beta}^{i}\right) d s \\
= & -\int_{0}^{t}\left(\sum_{j \neq i} \frac{R_{s, \beta}^{i}-R_{s, \beta}^{j}}{\left(\phi_{i}(s, x)-\phi_{j}(s, x)\right)^{2}}+\sum_{j \neq i} \frac{R_{s, k}^{i}+R_{s, k}^{j}}{\left(\phi_{i}(s, x)+\phi_{j}(s, x)\right)^{2}}\right. \\
& \left.+\nu \frac{R_{s, \beta}^{i}}{\phi_{i}(s, x)^{2}}+H_{s, \beta}^{i}\right) d s
\end{aligned}
$$

with the error terms

$$
\begin{aligned}
H_{s, \beta}^{i}=\beta[ & \sum_{j \neq i} \frac{\left(\left(X_{s, k}^{i}-\sqrt{\beta} \phi_{i}(s, x)\right)-\left(X_{s, k}^{j}-\sqrt{\beta} \phi_{j}(s, x)\right)\right)^{2}}{\left(\sqrt{\beta}\left(\phi_{i}(s, x)-\phi_{j}(s, x)\right)+D_{i, j}^{-}(s)\right)^{3}} \\
& +\sum_{j \neq i} \frac{\left(\left(X_{s, k}^{i}-\sqrt{\beta} \phi_{i}(s, x)\right)+\left(X_{s, k}^{j}-\sqrt{\beta} \phi_{j}(s, x)\right)\right)^{2}}{\left(\sqrt{\beta}\left(\phi_{i}(s, x)+\phi_{j}(s, x)\right)+D_{i, j}^{+}(s)\right)^{3}} \\
& \left.+\nu \frac{\left.\left(X_{s, k}^{j}-\sqrt{\beta} \phi_{j}(s, x)\right)\right)^{2}}{\left(\sqrt{\beta} \phi_{i}(s, x)+D_{i}(s)\right)^{3}}\right]
\end{aligned}
$$

where, by the Lagrange remainders in the 3 Taylor expansions above,

$$
\left|D_{i, j}^{ \pm}(s)\right| \leq\left|\left(X_{s, k}^{i}-\sqrt{\beta} \phi_{i}(s, x)\right) \pm\left(X_{s, k}^{j}-\sqrt{\beta} \phi_{j}(s, x)\right)\right|
$$

and

$$
\left|D_{i}(s)\right| \leq\left|X_{s, k}^{i}-\sqrt{\beta} \phi_{i}(s, x)\right| .
$$

By Theorem 3.1 the terms $\left|D_{i, j}^{ \pm}\right|,\left|D_{i}\right|$ can be bounded by some a.s. finite random variable $D$ independent of $i, j$, the sign, $s \in[0, t]$, and $\beta \geq \beta_{0}$ where $D$ depends on $x, y, t$. Therefore, for all $i=1, \ldots, N$,

$$
\left|H_{s, \beta}^{i}\right| \leq \frac{1}{\sqrt{\beta}} H \quad \text { for } \quad \beta \geq \beta_{0}, s \in[0, t]
$$

with some a.s. finite random variable $H$. In summary,

$$
R_{t, \beta}=-\int_{0}^{t}\left(A_{\nu}(s, x) R_{s, \beta}+H_{s, \beta}\right) d s, \quad R_{0, k}=0
$$


and thus, for suitable norms and all $u \in[0, t]$,

$$
\left\|R_{u, \beta}\right\| \leq A \int_{0}^{u}\left\|R_{s, \beta}\right\| d s+\frac{t \cdot\|H\|}{\sqrt{\beta}}
$$

with $A:=\sup _{s \in[0, t]}\left\|A_{\nu}(s, x)\right\|<\infty$. Hence, by the classical lemma of Gronwall,

$$
\left\|R_{u, \beta}\right\| \leq \frac{t\|H\|}{\sqrt{\beta}} e^{t A}
$$

for all $u \in[0, t]$. This yields the claim.

We next calculate the covariance matrix of $W_{t}$ for the special solution $\phi$ of (3.4). For this we introduce the matrices $A_{\nu}=\left(A_{\nu, i, j}\right)_{i, j} \in \mathbb{R}^{N \times N}$ with

$$
A_{\nu, i, j}:=\frac{1}{\left(y_{i}-y_{j}\right)^{2}}-\frac{1}{\left(y_{i}+y_{j}\right)^{2}}, \quad A_{\nu, i, i}:=\sum_{j \neq i}\left(\frac{-1}{\left(y_{i}-y_{j}\right)^{2}}-\frac{1}{\left(y_{i}+y_{j}\right)^{2}}\right)-\frac{\nu}{y_{i}^{2}}
$$

for $i, j=1, \ldots, N, i \neq j$ and $y$ as in (3.5). By [AV2], $E-2 A_{\nu}$ has the eigenvalues $2,4, \ldots, 2 N$ independent of $\nu$. With these notations we have:

Lemma 3.3. Let $\nu>0$. Assume that the Bessel process $\left(X_{t, k}\right)_{t \geq 0}$ of type $B$ with $k=(\nu \beta, \beta)$ starts in the point $\sqrt{\beta} \cdot c y+w$ in the interior of $C_{N}^{B}$ with $w \in \mathbb{R}, y$ as in (3.5), and $c>0$. Then, the covariance matrices $\Sigma_{\nu, t} \in \mathbb{R}^{N \times N}$ for $t>0$ of the limit Gaussian process $\left(W_{t}\right)_{t \geq 0}$ are given by

$$
\Sigma_{\nu, t}=\left(t+c^{2}\right)\left(E-2 A_{\nu}\right)^{-1}\left(E-e^{\ln \frac{c^{2}}{t+c^{2}}\left(E-2 A_{\nu}\right)}\right)
$$

with eigenvalues $\lambda_{k}^{B}(t, c)=\frac{1}{2 k} \frac{\left(t+c^{2}\right)^{2 k}-c^{4 k}}{\left(t+c^{2}\right)^{2 k-1}}(k=1, \ldots, N)$.

Proof. For the special case $\phi(s, c y)=\sqrt{s+c^{2}} y$ with $c>0$ and the vector $y$ in (3.5), the matrix function $A_{\nu}(s, c z)$ has the form $A_{\nu}(s, c y)=\frac{1}{s+c^{2}} A_{\nu}$. Hence,

$$
W_{t}=e^{\left(\ln \left(t+c^{2}\right)-\ln c^{2}\right) A_{\nu}} \int_{0}^{t} e^{\left(-\ln \left(s+c^{2}\right)+\ln c^{2}\right) A_{\nu}} d B_{s} \quad(t \geq 0) .
$$

Since $A_{\nu}$ is real and symmetric with eigenvalues $2,4, \ldots, 2 N$, we may write $A_{\nu}$ as $A_{\nu}=U D U^{t}$ with an orthogonal matrix $U$ and the diagonal matrix

$$
D=\operatorname{diag}\left(d_{1}, \ldots d_{N}\right):=\operatorname{diag}(-1 / 2,-3 / 2, \ldots,(-2 N+1) / 2) .
$$

Hence,

$$
W_{t}=U \int_{0}^{t} \operatorname{diag}\left(\left(\frac{t+c^{2}}{s+c^{2}}\right)^{d_{1}}, \ldots,\left(\frac{t+c^{2}}{s+c^{2}}\right)^{d_{N}}\right) d \tilde{B}_{s} \cdot U^{t}
$$

with the rotated Brownian motion $\left(\tilde{B}_{t}:=U^{t} B_{t} U\right)_{t \geq 0}$. Now similar arguments as in the proof of Lemma 2.6 yield the desired form of the covariance matrix.

Remark 3.4. The eigenvalues of $\Sigma_{t}$ in the cases $A_{2 N-1}$ and $B_{N}$ are related by

$$
\lambda_{i}^{B}(t, c)=\lambda_{2 i}^{A}(t, c \cdot \sqrt{2}) \quad(i=1, \ldots, N)
$$

independent of $\nu$. This seems to be connected in some way with the formula $H_{2 N}(x)=$ const. $(N) \cdot L_{N}^{(-1 / 2)}\left(x^{2}\right)$ for $\nu=1 / 2$.

All preceding results hold for $\nu>0$. We show in Section 4 that most results are also valid for $\nu=0$ with some modifications. 


\section{The Root System $D_{N}$}

We now briefly study limit theorems for Bessel processes of type $D_{N}$. We recapitulate that the associated closed Weyl chamber is

$$
C_{N}^{D}=\left\{x \in \mathbb{R}^{N}: \quad x_{1} \geq \ldots \geq x_{N-1} \geq\left|x_{N}\right|\right\},
$$

i.e., $C_{N}^{D}$ is a doubling of $C_{N}^{B}$ w.r.t. the last coordinate. We have a one-dimensional multiplicity $k \geq 0$, and the SDE (1.6) for the processes $\left(X_{t, k}\right)_{t \geq 0}$ of type $D$ is

$$
d X_{t, k}^{i}=d B_{t}^{i}+k \sum_{j \neq i}\left(\frac{1}{X_{t, k}^{i}-X_{t, k}^{j}}+\frac{1}{X_{t, k}^{i}+X_{t, k}^{j}}\right) d t
$$

for $i=1, \ldots, N$ with an $N$-dimensional Brownian motion $\left(B_{t}^{1}, \ldots, B_{t}^{N}\right)_{t \geq 0}$. The renormalized processes $\left(\tilde{X}_{t, k}:=X_{t, k} / \sqrt{k}\right)_{t \geq 0}$ satisfy

$$
d \tilde{X}_{t, k}^{i}=\frac{1}{\sqrt{k}} d B_{t}^{i}+\sum_{j \neq i}\left(\frac{1}{\tilde{X}_{t, k}^{i}-\tilde{X}_{t, k}^{j}}+\frac{1}{\tilde{X}_{t, k}^{i}+\tilde{X}_{t, k}^{j}}\right) d t \quad(i=1, \ldots, N) .
$$

For $k=\infty$ we have from Lemma 4.1 of [AV1] that for each starting point $x_{0}$ in the interior of $C_{N}^{D}$, the ODE

with

$$
\frac{d x}{d t}(t)=H(x(t))
$$

$$
H(x):=\left(\begin{array}{c}
\sum_{j \neq 1}\left(\frac{1}{x_{1}-x_{j}}+\frac{1}{x_{1}+x_{j}}\right) \\
\vdots \\
\sum_{j \neq N}\left(\frac{1}{x_{N}-x_{j}}+\frac{1}{x_{N}+x_{j}}\right)
\end{array}\right)
$$

has a unique solution $\phi\left(t, x_{0}\right)$ in the interior of $C_{N}^{D}$ for $t \geq 0$. Again, as in Section 4 of AV1, a special solution associated to the zeros of Laguerre polynomials may be derived. Using the representation

$$
L_{N}^{(\alpha)}(x):=\sum_{k=0}^{N}\left(\begin{array}{c}
N+\alpha \\
N-k
\end{array}\right) \frac{(-x)^{k}}{k !} \quad(\alpha \in \mathbb{R}, N \in \mathbb{N})
$$

of the Laguerre polynomials according to (5.1.6) of Szegö $[\underline{\mathrm{S}}$, we form the polynomial $L_{N}^{(-1)}$ of order $N \geq 1$ where, by (5.2.1) of [S],

$$
L_{N}^{(-1)}(x)=-\frac{x}{N} L_{N-1}^{(1)}(x) .
$$

We now denote the $N$ zeros of $L_{N}^{(-1)}$ by $z_{1}>\ldots>z_{N}=0$ and define

$$
2 \cdot\left(z_{1}^{(1)}, \ldots, z_{N-1}^{(1)}, 0\right)=\left(r_{1}^{2}, \ldots, r_{N}^{2}\right) .
$$

Then we obtain for the starting points $c r$ with $c>0$, the particular solutions

$$
\phi(t, c \cdot r)=\sqrt{t+c^{2}} \cdot r
$$

of the ODE (4.3); cf. AV1.

Again, the solutions (4.6) are attracting in some way. Moreover, as in the preceding sections, we have

$$
\|\phi(t, x)\|^{2}=2 N(N-1) t+\|x\|^{2} \quad(t \geq 0) .
$$

Beside the particular solutions given by (4.6) we have the following observation which fits with Eq. (4.4). 
Lemma 4.1. Let $x$ be a point in the interior of $C_{N}^{D}$ with $x_{N}=0$. Then the associated solution of the ODE (4.3) satisfies $\phi(t, x)_{N}=0$ for all $t$, and the first $N-1$ components $\left(\phi(t, x)_{1}, \ldots, \phi(t, x)_{N-1}\right)$ solve the $O D E$ of the $B$-case in (3.3) with dimension $N-1$ and $\nu=2$.

Proof. If $x_{N}=0$, then by the ODE in (4.3), $\frac{d}{d t} \phi(t, x)_{N}=0$.

We next recapitulate the following strong limit law; see Theorem 5.5 of AV1:

Theorem 4.2. Let $x$ be a point in the interior of $C_{N}^{D}$, and $y \in \mathbb{R}^{N}$. Let $k \geq 1 / 2$ with $\sqrt{k} \cdot x+y$ in the interior of $C_{N}^{B}$ for $k \geq k_{0}$. For $k \geq k_{0}$, consider the Bessel processes $\left(X_{t, k}\right)_{t \geq 0}$ of type $D_{N}$ starting in $\sqrt{k} \cdot x+y$. Then, for all $t>0$,

$$
\sup _{0 \leq s \leq t, k \geq k_{0}}\left\|X_{s, k}-\sqrt{k} \phi(s, x)\right\|<\infty \quad \text { a.s.. }
$$

In particular, $X_{t, k} / \sqrt{k} \rightarrow \phi(t, x)$ for $k \rightarrow \infty$ locally uniformly in $t$ a.s..

We now turn to an associated functional CLT. We fix some $x$ in the interior of $C_{N}^{D}$ and consider the associated solution $\phi(t, x)$. We also introduce an $N$-dimensional process $\left(W_{t}\right)_{t \geq 0}$ as the unique solution of the inhomogeneous linear SDE

$$
d W_{t}^{i}=d B_{t}^{i}+\sum_{j \neq i}\left(\frac{W_{t}^{j}-W_{t}^{i}}{\left(\phi_{i}(t, x)-\phi_{j}(t, x)\right)^{2}}-\frac{W_{t}^{j}+W_{t}^{i}}{\left(\phi_{i}(t, x)+\phi_{j}(t, x)\right)^{2}}\right) d t
$$

for $i=1, \ldots, N$ with initial condition $W_{0}=0$. (4.8) may be written as

$$
d W_{t}=d B_{t}+A(t, x) W_{t} d t
$$

with the matrix $A(t, x) \in \mathbb{R}^{N \times N}$ with

$$
\begin{aligned}
A(t, x)_{i, j} & :=\frac{1}{\left(\phi_{i}(t, x)-\phi_{j}(t, x)\right)^{2}}-\frac{1}{\left(\phi_{i}(t, x)+\phi_{j}(t, x)\right)^{2}} \quad(i \neq j), \\
A(t, x)_{i, i} & :=\sum_{j \neq i}\left(\frac{-1}{\left(\phi_{i}(t, x)-\phi_{j}(t, x)\right)^{2}}-\frac{1}{\left(\phi_{i}(t, x)+\phi_{j}(t, x)\right)^{2}}\right)
\end{aligned}
$$

for $i, j=1, \ldots, N$. The process $\left(W_{t}\right)_{t \geq 0}$ is Gaussian and given by

$$
W_{t}=e^{\int_{0}^{t} A(s, x) d s} \int_{0}^{t} e^{-\int_{0}^{s} A(u, x) d u} d B_{s} \quad(t \geq 0) .
$$

It is related to the Bessel processes $\left(X_{t, k}\right)_{t \geq 0}$ of type $D$ by the following result. As the proof is completely analogous to that of Theorem 3.2. we omit the proof.

Theorem 4.3. Let $x$ be a point in the interior of $C_{N}^{D}$ and $y \in \mathbb{R}^{N}$. Let $k_{0} \geq 1 / 2$ such that $\sqrt{k} \cdot x+y$ is in the interior of $C_{N}^{D}$ for $k \geq k_{0}$. For $k \geq k_{0}$ consider the Bessel processes $\left(X_{t, k}\right)_{t \geq 0}$ starting at $\sqrt{k} \cdot x+y$. Then, for all $t>0$,

$$
\sup _{0 \leq s \leq t, k \geq k_{0}} \sqrt{k} \cdot\left\|X_{s, k}-\sqrt{k} \phi(s, x)-W_{s}\right\|<\infty \quad \text { a.s., }
$$

i.e., $X_{s, k}-\sqrt{k} \phi(s, x) \rightarrow W_{s}$ locally uniformly in $s$ a.s. with rate $O(1 / \sqrt{k})$.

Remark 4.4. Consider the Bessel processes $\left(X_{t, k}\right)_{t \geq 0}$ of Theorem 4.3 which start in $\sqrt{k} \cdot x$ for $x$ in the interior of $C_{N}^{D}$ with $x_{N}=0$. Then, by Lemma 4.1, $\phi(t, x)_{N}=0$ for all $t \geq 0$, and the matrix function $A$ from (4.10) satisfies $A(t, x)_{N, N}=0$. 
We next calculate the covariance matrix of $W_{t}$ for the special solution $\phi$ in (4.6). For this we introduce the matrix $A \in \mathbb{R}^{N \times N}$ with

$$
A_{i, j}:=\frac{1}{\left(r_{i}-r_{j}\right)^{2}}-\frac{1}{\left(r_{i}+r_{j}\right)^{2}}, \quad A_{i, i}:=\sum_{j \neq i}\left(\frac{-1}{\left(r_{i}-r_{j}\right)^{2}}-\frac{1}{\left(r_{i}+r_{j}\right)^{2}}\right)
$$

for $i, j=1, \ldots, N, i \neq j$ and the vector $r$ as in (4.5). By [AV2, $E-2 A$ has the eigenvalues $2,4, \ldots, 2 N$. We obtain the following result. As its proof is again analog to that of Lemma 3.3, we skip the proof.

Lemma 4.5. Assume that the Bessel processes $\left(X_{t, k}\right)_{t \geq 0}$ of type $D_{N}$ start in the points $\sqrt{k} \cdot c r+w$ in the interior of $C_{N}^{D}$ with $w \in \mathbb{R}^{N}, r$ given in $\sqrt{4.5}$ ), and $c>0$. Then, the covariance matrices $\Sigma_{t} \in \mathbb{R}^{N \times N}$ for $t>0$ of the limit Gaussian process $\left(W_{t}\right)_{t \geq 0}$ are

$$
\Sigma_{t}=\left(t+c^{2}\right)(E-2 A)^{-1}\left(E-e^{\ln \frac{c^{2}}{t+c^{2}}(E-2 A)}\right) .
$$

Remark 4.6. Let $x$ be in the interior of $C_{N}^{D}$ with $x_{N}=0$. Then, by Lemma 4.1. $A(t, x)_{N, j}=A(t, x)_{j, N}=0$ for $j \neq N$ and $t>0$ in (4.10). Hence, by Eq. (4.11), that the $N$-th component $\left(W_{t}^{(N)}\right)_{t \geq 0}$ of $\left(W_{t}\right)_{t \geq 0}$ is independent from $\left(W_{t}^{(1)}, \ldots, W_{t}^{(N-1)}\right)_{t \geq 0}$. This appears in particular in the setting of Lemma 4.5.

\section{Further limit theorems For the CASE B}

Section 4 is closely related to limit results for Bessel processes of type B for $\left(k_{1}, k_{2}\right)=(0, k)$ for $k \rightarrow \infty$ which was excluded in Section 3. To explain this, we recapitulate some facts from [AV1, [V]. Let $\left(X_{t, k}^{D}\right)_{t \geq 0}$ be a Bessel process of type D with multiplicity $k \geq 0$ on $C_{N}^{D}$ with starting point $x$ in the interior of $C_{N}^{D}$. It follows from the generator of the associated semigroup that then the process $\left(X_{t, k}^{B}\right)_{t \geq 0}$ with

$$
X_{t, k}^{B, i}:=X_{t, k}^{D, i} \quad(i=1, \ldots, N-1), \quad X_{t, k}^{B, N}:=\left|X_{t, k}^{D, N}\right|
$$

is a Bessel process of type B with the multiplicity $\left(k_{1}, k_{2}\right):=(0, k)$ and starting point $\left(x_{1}, \ldots, x_{N-1},\left|x_{N}\right|\right) \in C_{N}^{B}$ with $x_{1}>\ldots>x_{N-1}>\left|x_{N}\right| \geq 0$. Notice that $\left(X_{t, k}^{B}\right)_{t \geq 0}$ is a diffusion with reflecting boundary where the boundary parts with the $N$-th coordinate equal to zero are attained.

We now translate the results of Section 4. For this we consider the solutions $\phi(t, x)$ of the ODE (4.3) in the following two particular cases for $t \geq 0$ :

(1) If $x$ is in the interior of $C_{N}^{B}$, then $\phi(t, x)$ is also in the interior of $C_{N}^{B}$.

(2) If $x_{1}>\ldots>x_{N-1}>x_{N}=0$, then $\phi(t, x)_{1}>\ldots>\phi(t, x)_{N-1}>$ $\phi(t, x)_{N}=0$.

Case (2) appears in particular for $\phi(t, x)=\sqrt{t+c^{2}} \cdot r$ for $c>0$ and the vector $r$ as in (4.6) with $r_{N}=0$.

Theorem 4.2 now reads as follows for the B-case with $\left(k_{1}, k_{2}\right)=(0, k)$ for $k \rightarrow \infty$ :

Theorem 5.1. Let $x$ be as described above in (1) or (2). For $k \geq 1 / 2$, consider the Bessel processes $\left(X_{t,(0, k)}\right)_{t \geq 0}$ of type $B_{N}$ starting in $\sqrt{k} \cdot x$. Then, for $t>0$,

$$
\sup _{0 \leq s \leq t, k \geq 1 / 2}\left\|X_{s, k}-\sqrt{k} \phi(s, x)\right\|<\infty \quad \text { a.s.. }
$$

We next consider the Gaussian processes $\left(W_{t}\right)_{t \geq 0}$ of Eq. (4.11). Theorem4.3 now leads to functional CLTs where the cases (1) and (2) have to be treated separately for geometric reasons. For the case (1) we have the following result: 
Theorem 5.2. Let $x$ be a point in the interior of $C_{N}^{B}$. For $k \geq 1 / 2$ consider Bessel processes $\left(X_{t,(0, k)}\right)_{t \geq 0}$ of type $B$ starting at $\sqrt{k} \cdot x$. Then, for all $t>0$,

$$
\sup _{0 \leq s \leq t, k \geq k_{0}} \sqrt{k} \cdot\left\|X_{s,(0, k)}-\sqrt{k} \phi(s, x)-W_{s}\right\|<\infty \quad \text { a.s.. }
$$

Proof. As $x$ is in the interior of $C_{N}^{B}$, we obtain that for each $t>0$ and almost all $\omega \in \Omega$, the path $\left(\sqrt{k} \phi(s, x)-W_{s}(\omega)\right)_{s \in[0, t]}$ is arbitrarily far away from the boundary of $C_{N}^{B}$ whenever $k$ is sufficiently large. This, the connection between the $\mathrm{D}$ - and B-case, and Theorem 4.3 thus lead to the theorem.

Theorem 5.2 corresponds to Theorem 3.2 for $\nu=0$. We next turn to case (2):

Theorem 5.3. Let $x \in C_{N}^{B}$ with $x_{1}>\ldots>x_{N-1}>x_{N}=0$. For $k \geq 1 / 2$ consider Bessel processes $\left(X_{t,(0, k)}\right)_{t \geq 0}$ of type $B$ starting at $\sqrt{k} \cdot x$. Then, for the process $\left(\tilde{W}_{t}:=\left(W_{t}^{(1)}, \ldots, W_{t}^{(N-1)},\left|W_{t}^{(N)}\right|\right)\right)_{t \geq 0}$, and all $t>0$,

$$
\sup _{0 \leq s \leq t, k \geq k_{0}} \sqrt{k} \cdot\left\|X_{s,(0, k)}-\sqrt{k} \phi(s, x)-\tilde{W}_{s}\right\|<\infty \quad \text { a.s.. }
$$

Proof. This follows immediately from Theorem 4.3 , the connection between the Dand B-case, and from ||$a|-| b|| \leq|a-b|$ for $a, b \in \mathbb{R}$.

For the process $\left(\tilde{W}_{t}\right)_{t \geq 0}$, the first $N-1$ components form a Gaussian process which is independent from $\left(\left|W_{t}^{(N)}\right|\right)_{t \geq 0}$ by Remark 4.6. The variables $\left|W_{t}^{(N)}\right|$ are one-sided normal distributed. Therefore, Theorems 5.2 and 5.3 lead to a discontinuity (or phase transition) in the limit depending on the starting points here.

\section{Extensions to multi-dimensional Bessel processes With an ADDITIONAL ORNSTEIN-UHLENBECK COMPONENT}

In this section we consider an extension of our previous models by adding an additional drift coefficient of the form $-\lambda x, \lambda \in \mathbb{R}$, i.e., a component as in a classical Ornstein-Uhlenbeck setting

$$
d Y_{t, k}=d B_{t}+\left(\frac{1}{2}\left(\nabla\left(\ln w_{k}\right)\right)\left(Y_{t, k}\right)-\lambda Y_{t, k}\right) d t .
$$

If $\lambda>0$, we obtain a mean reverting ergodic process with speed of mean-reversion $\lambda$. For $\lambda \leq 0$ the process is non-ergodic. For $N=1$ and $\lambda>0$ the squared process is the well-known Cox-Ingersoll-Ross process from mathematical finance.

We derive the results for the root system $A_{N-1}$ only as the same technique also holds for the other root systems. We consider processes $\left(Y_{t, k}\right)_{t \geq 0}$ of type $A_{N-1}$ as solutions of

$$
d Y_{t, k}^{i}=d B_{t}^{i}+\left(k \sum_{j \neq i} \frac{1}{Y_{t, k}^{i}-Y_{t, k}^{j}}-\lambda Y_{t, k}^{i}\right) d t \quad(i=1, \ldots, N) .
$$

with an $N$-dimensional Brownian motion $\left(B_{t}^{1}, \ldots, B_{t}^{N}\right)_{t \geq 0}$. Itô's formula and a time-change argument show that $Y$ is a space-time transformation of the original $X$ (with $\lambda=0$ ), namely

$$
Y_{t, k}=e^{-\lambda t} X_{\frac{e^{2 \lambda t}-1}{2 \lambda}, k}
$$

For a proof based on the generators cf. RV1. A similar relation holds for the associated ODEs. 
Lemma 6.1. Let $\phi(t, x)$ be a solution of the dynamical system $\frac{d x}{d t}(t)=H(x(t))$ with starting point $x$ in the interior of $C_{N}^{A}$ as in Section 2. Then

$$
\phi\left(\frac{1-e^{-2 \lambda t}}{2 \lambda}, e^{-\lambda t} x\right)
$$

solves the $O D E \frac{d x}{d t}(t)=H(x(t))-\lambda x(t)$ with starting point $x$.

Proof. This follows from the space-time homogeneity in Remark 2.3.

With the techniques in Theorem 2.1 and Theorem 2.2 we obtain a functional CLT for $\left(Y_{t, k}\right)_{t \geq 0}$ :

$$
\sqrt{k}\left(\frac{Y_{t, k}}{\sqrt{k}}-\phi\left(\frac{1-e^{-2 \lambda t}}{2 \lambda}, e^{-\lambda t} x\right)\right) \longrightarrow W_{t}
$$

for $k \rightarrow \infty$ locally uniformly in $t$ a.s. with rate $O(1 / \sqrt{k})$, where

$$
d W_{t}=d B_{t}+A^{\lambda}(t, x) W_{t} d t
$$

with the matrices $A^{\lambda}(t, x) \in \mathbb{R}^{N \times N}$ with

$$
\begin{gathered}
A^{\lambda}(t, x)_{i, j}:=\frac{1}{\left(\phi_{i}\left(\frac{1-e^{-2 \lambda t}}{2 \lambda}, e^{-\lambda t} x\right)-\phi_{j}\left(\frac{1-e^{-2 \lambda t}}{2 \lambda}, e^{-\lambda t} x\right)\right)^{2}}, \\
A^{\lambda}(t, x)_{i, i}:=-\sum_{j \neq i} \frac{1}{\left(\phi_{i}\left(\frac{1-e^{-2 \lambda t}}{2 \lambda}, e^{-\lambda t} x\right)-\phi_{j}\left(\frac{1-e^{-2 \lambda t}}{2 \lambda}, e^{-\lambda t} x\right)\right)^{2}}-\lambda
\end{gathered}
$$

for $i, j=1, \ldots, N, i \neq j$ and with initial condition $W_{0}=0$. The process $\left(W_{t}\right)_{t \geq 0}$ admits the explicit representation

$$
W_{t}=e^{\int_{0}^{t} A^{\lambda}(s, x) d s} \int_{0}^{t} e^{-\int_{0}^{s} A^{\lambda}(u, x) d u} d B_{s} \quad(t \geq 0) .
$$

Note that due to the constant term in the diagonal of $A^{\lambda}(t, x)$ for $\lambda \neq 0$, we obtain a linear time-dependence in the exponential of the matrix exponential which dominates the long-term behaviour of the covariance matrix. In particular:

Lemma 6.2. Assume that $\left(Y_{t, k}\right)_{t \geq 0}$ starts in the interior of $C_{N}^{A}$ in $\sqrt{k} \cdot c z+y$ with $y \in \mathbb{R}, z$ as in 2.5 and $c>0$. Then the covariance matrices $\Sigma_{t}^{\lambda} \in \mathbb{R}^{N \times N}$ for $t>0$ of the limit process $\left(W_{t}\right)_{t \geq 0}$ are given by

$$
\Sigma_{t}^{\lambda}=\frac{1+e^{-2 \lambda t}\left(\lambda c^{2}-1\right)}{2 \lambda}(E-A)^{-1}\left(E-e^{\ln \frac{\lambda c^{2}}{e^{2 \lambda t}-1+\lambda c^{2}}(E-A)}\right),
$$

where $A$ is defined by (2.15).

Proof. For the special starting points $c z$ we obtain the special solution

$$
\phi\left(\frac{1-e^{-2 \lambda s}}{2 \lambda}, e^{-\lambda s} c z\right)=e^{-\lambda s} \sqrt{\frac{e^{2 \lambda s}-1}{\lambda}+c^{2} z} .
$$

Hence the matrix function $A^{\lambda}(s, c z)$ has the simple form with the same timedependence for each entry

$$
A^{\lambda}(s, c z)=\frac{\lambda e^{2 \lambda s}}{e^{2 \lambda s}-1+\lambda c^{2}} A-\operatorname{diag}(\lambda s, \cdots, \lambda s),
$$

where $A$ is given by (2.15). This yields the process

$$
W_{t}=\int_{0}^{t} e^{(t-s) \operatorname{diag}(-\lambda, \cdots,-\lambda)+\ln \left(\frac{e^{2 \lambda t}-1+\lambda c^{2}}{e^{2 \lambda s}-1+\lambda c^{2}}\right) A} d B_{s} \quad(t \geq 0) .
$$


Now we may proceed as in Lemma 2.6 to calculate the covariance matrix.

Remark 6.3. The long-term behaviour of the covariance matrix is inherited by the long-term behaviour of $Y$. In the ergodic case for $Y$, i.e. $\lambda>0$, we obtain $\lim _{t \rightarrow \infty} \Sigma_{t}^{\lambda}=\frac{1}{2 \lambda}(E-A)^{-1}$. For $\lambda<0$ we need an exponential scaling

$$
\lim _{t \rightarrow \infty} e^{2 \lambda t} \Sigma_{t}^{\lambda}=\frac{\lambda c^{2}-1}{2 \lambda}(E-A)^{-1}\left(E-e^{\ln \left(\frac{\lambda c^{2}}{\lambda c^{2}-1}\right)(E-A)}\right) .
$$

\section{REFERENCES}

[AG] R. Allez, A. Guionnet, A diffusive matrix model for invariant $\beta$-ensembles. Electron. J. Probab. 18 (2013), 1-30.

[AKM1] S. Andraus, M. Katori, S. Miyashita, Interacting particles on the line and Dunkl intertwining operator of type A: Application to the freezing regime. J. Phys. A: Math. Theor. 45 (2012) 395201.

[AKM2] S. Andraus, M. Katori, S. Miyashita, Two limiting regimes of interacting Bessel processes. J. Phys. A: Math. Theor. 47 (2014) 235201.

[AM] S. Andraus, S. Miyashita, Two-step asymptotics of scaled Dunkl processes. J. Math. Phys. $56(2015) 103302$.

[AV1] S. Andraus, M. Voit, Limit theorems for multivariate Bessel processes in the freezing regime. Stoch. Proc. Appl. 129 (2019), 4771-4790.

[AV2] S. Andraus, M. Voit, Central limit theorems for multivariate Bessel processes in the freezing regime II: The covariance matrices. J. Approx. Theory 246 (2019), 6584.

[A] J.-P. Anker. An introduction to Dunkl theory and its analytic aspects. In: G. Filipuk, Y. Haraoka, S. Michalik. Analytic, Algebraic and Geometric Aspects of Differential Equations, Birkhäuser, pp.3-58, 2017.

[BF] T.H. Baker, P.J. Forrester, The Calogero-Sutherland model and generalized classical polynomials. Comm. Math. Phys. 188 (1997), 175-216.

[CGY] O. Chybiryakov, L. Gallardo, M. Yor, Dunkl processes and their radial parts relative to a root system. In: P. Graczyk et al. (eds.), Harmonic and stochastic analysis of Dunkl processes. Hermann, Paris 2008.

[Dem] N. Demni, Generalized Bessel function of type D. SIGMA 4 (2008), 075, 7 pages, arXiv:0811.0507.

[DF] P. Desrosiers, P. Forrester, Hermite and Laguerre $\beta$-ensembles: Asymptotic corrections to the eigenvalue density. Nuclear Physics B 743 (2006), 307-332.

[DV] J.F. van Diejen, L. Vinet, Calogero-Sutherland-Moser Models. CRM Series in Mathematical Physics, Springer, Berlin, 2000.

[DE1] I. Dumitriu, A. Edelman, Matrix models for beta-ensembles. J. Math. Phys. 43 (2002), 5830-5847.

[DE2] I. Dumitriu, A. Edelman, Eigenvalues of Hermite and Laguerre ensembles: large beta asymptotics, Ann. Inst. Henri Poincare (B) 41 (2005), 1083-1099.

[GY] L. Gallardo, M. Yor, Some remarkable properties of the Dunkl martingale. In: Seminaire de Probabilites XXXIX, pp. 337-356, dedicated to P.A. Meyer, vol. 1874, Lecture Notes in Mathematics, Springer, Berlin, 2006.

[GM] P. Graczyk, J. Malecki, Strong solutions of non-colliding particle systems. Electron. J. Probab. 19 (2014), 21 pp.

[HP] D. Holcomb, E. Paquette, Tridigonal models for Dyson Brownian motion. Preprint 2017, arXiv 1707.02700v1.

[P] P.E. Protter, Stochastic Integration and Differential Equations. A New Approach. Springer, Berlin, 2003.

[R1] M. Rösler, Generalized Hermite polynomials and the heat equation for Dunkl operators. Comm. Math. Phys. 192 (1998), 519-542.

[R2] M. Rösler, Dunkl operators: Theory and applications. In: Orthogonal polynomials and special functions, Leuven 2002, Lecture Notes in Math. 1817 (2003), 93-135.

[RV1] M. Rösler, M. Voit, Markov processes related with Dunkl operators. Adv. Appl. Math. 21 (1998) 575-643. 
[RV2] M. Rösler, M. Voit, Dunkl theory, convolution algebras, and related Markov processes. In: P. Graczyk et al. (eds.), Harmonic and stochastic analysis of Dunkl processes. Hermann, Paris 2008.

[Sch] B. Schapira, The Heckman-Opdam Markov processes. Probab. Theory Rel. Fields 138 (2007), 495-519.

[S] G. Szegö, Orthogonal Polynomials. Colloquium Publications (American Mathematical Society), Providence, 1939

[V] M. Voit, Central limit theorems for multivariate Bessel processes in the freezing regime. $J$. Approx. Theory 239 (2019), 210-231.

[VW] M. Voit, J.H.C. Woerner, The differential equations associated with Calogero-MoserSutherland particle models in the freezing regime. Preprint 2019.

Fakultät Mathematik, Technische Universität Dortmund, Vogelpothsweg 87, D-44221 Dortmund, Germany

E-mail address: michael.voit@math.tu-dortmund.de, jeannette.woerner@math.tu-dortmund.de 